\title{
The IAEA/WHO TLD Audit Program. The results of the TLD postal dosimetry audits in the National Centre for Radiotherapy and Nuclear Medicine Department, Korle-Bu, Accra (1998 to 2012)
}

Theophilus. A. Sackey ${ }^{1,2}$, Issahaku Shirazu1, Mark Pokoo-Aikinss ${ }^{1}$, Samuel Nii Adu Tagoe², Elvis K. Tiburu ${ }^{3}$

${ }^{1}$ Ghana Atomic Energy Commission, Kwabenya-Accra

${ }^{2}$ National Centre for Radiotherapy and Nuclear Medicine Department, Accra.

${ }^{3}$ Department of Biomedical Engineering, University of Ghana, Legon-Accra

\begin{abstract}
During therapeutic procedures, the patient is always protected by the quality assurance program legitimately required for medical exposures. This comprehensive quality assurance programs, or quality management systems, include all components of radiation therapy practice, and has proven to be necessary in reducing the likelihood of dose misadministration. The aim of this paper is to gives a summary of various studies performed during the period of 15 years (1998-2012), and provides the results of a survey conducted by the IAEA. The methodology involve checking the beam output with its dosimeters, and to irradiate two TLD capsules in sequence in such a way that the irradiation time or monitor units calculation would deliver 200 cGy to a 'tumour' whose centre (the TLD capsule) is at $5 \mathrm{~cm}$ depth. Here, the irradiations were done in reference conditions using the agency's Perspex holder, and the delivered dose is not a 'given dose' at the depth of maximum dose, but a dose at depth. The results of this study demonstrate that the QUATRO audit programme is a very useful tool for reviewing practices at our Centre, and shown beyond doubt to be effective in assuring the quality of dose determination at the Centre. Our study further indicates that it is possible to have the dose determination within acceptance limits by implementing correct procedures and cautiously performing dose calculations and measurements
\end{abstract}

Keywords : TLD, QUATRO, IAEA, Quality Assurance, SSDL

\section{INTRODUCTION}

In radiotherapy, the ultimate aim is to ensure that the prescribed dose is rightly given to the tumour, whilst keeping the dose to surrounding healthy tissues and critical organs to a minimal dose. During therapeutic procedures, the patient is always protected by the quality assurance program legitimately required for medical exposures (Waligórski \& Lesiak, 2001). This comprehensive quality assurance (QA) programs, or quality management systems, include all components of radiation therapy practice, and has proven to be necessary in reducing the likelihood of dose misadministration (WHO, 1988) (ICRP, 2000) (IAEA, 2000). In light of this, the beam calibration audit which determines the doses in reference conditions, is recognized as an essential element of QA programs in reducing overall uncertainty of the radiotherapy procedure, and that certain scientific institutions, such as the Secondary Standard Dosimetry Laboratories (SSDL) are usually competent in such activity (IAEA/WHO, 2001) (Groth, et al., 2001). The ultimate purpose of the external dosimetry quality audits in radiation therapy is to improve the accuracy and consistency of clinical dosimetry in radiotherapy centres worldwide, and to prevent or 
reduce the probability of errors and accidents (Izewska, et al., 2007).

Several radiotherapy accidents which could have been prevented, have been given on overexposure of patients. Notably among these is the Costa Rica incidence which occurred in 1996 (IAEA, 1998). In such incident, the absorbed dose rate of the new source was underestimated, resulting in treatment times being overestimated by $66 \%$, and leading overexposures involving 115 patients. Another radiotherapy accident which occurred at the National Oncology Institute (Instituto Oncológico Nacional, ION), Panama (Borrás, 2006), is also reported. The incident involved the overexposure of 28 radiation therapy patients at the ION in late 2000 and early 2001. Also, at another hospital clinic located in Zaragoza, Spain, between the dates of December 10 and December 20, 1990, at least 27 patients who were receiving radiotherapy for cancer were accidentally exposed to high levels of radiation, which resulted in the deaths of 11 patients, and severe injuries to the others (IAEA/WHO, 1997). With these and other reported cases of unintended exposures, The IAEA/WHO TLD postal audit program has been an important tool in reducing radiotherapy accidents.

The IAEA/WHO TLD postal audit program is a collaboration between the IAEA and the WHO. It was implemented to verify the calibration of radiotherapy beams in developing countries. At the start, the IAEA/WHO TLD postal dose quality audit had been established for ${ }^{60} \mathrm{Co}$ radiotherapy units. However, since 1991, the introduction of new and advanced therapy techniques compelled the IAEA and WHO to include audits of high-energy X-rays produced by clinical accelerators (IAEA/WHO, 1997) (Izewska \& Andreo, 2000) (Izewska, et al., 2004) (Izewska, et al., 2002).

In the IAEA/WHO TLD postal dose quality audit, the $\mathrm{WHO}$ is responsible for the distribution and collection of the TLDs to and from radiotherapy institutions using WHO national or regional affiliated centres of coordinating the distribution of the dosimeters, while the IAEA's Dosimetry and Medical Radiation Physics Section is responsible for the technical aspects of the TLD system, reference irradiations and evaluation of the TLDs (IAEA/WHO, 1997). The maximum acceptable discrepancy between the dose stated by the centre and the dose evaluated by the IAEA is $\pm 5 \%$. In cases where discrepancies are larger, an additional audit is performed. If the problem persists after the second audit, the IAEA steps-in to offer assistance to the particular centre, this approach is taken by IAEA in order to resolve the problem.

\section{OBJECTIVES}

The aim of this paper is to gives a summary of various studies performed during the period of 15 years (1998-2012), and provides the results of a survey conducted by the IAEA. The survey had the aim of checking the dose delivered by the radiotherapy unit of the National Centre for Radiotherapy and Nuclear Medicine Department. The testing for the accuracy and consistency of basic dosimetric - calibration of radiotherapy beams with an ionization chamber, in reference conditions.

\section{MATERIAL AND METHODS}

In order to detect possible errors in the dose calculations, and to check whether the dosimetry protocol was properly followed, the National Centre for Radiotherapy and Nuclear Medicine Department, Korle-Bu was provided a data sheet. The provision of the data sheet was aimed at retrieving from the centre, the specifications of the treatment unit and measuring instruments, the method used for absorbed dose to water determination, coefficients and factors applied, results of dose measurements, and the details concerning the irradiation of TLD capsules. Additionally, the centre was provided with an 
instruction sheet that described the whole irradiation procedure.

In every investigation, the centre was asked to check the beam output with its dosimeters, and to irradiate two TLD capsules in sequence in such a way that the irradiation time or monitor units calculation would deliver 200 cGy to a 'tumour' whose centre (the TLD capsule) is at $5 \mathrm{~cm}$ depth. Here, the irradiations were done in reference conditions using the agency's Perspex holder, and the delivered dose is not a 'given dose' at the depth of maximum dose, but a dose at depth (as in Figure 1).

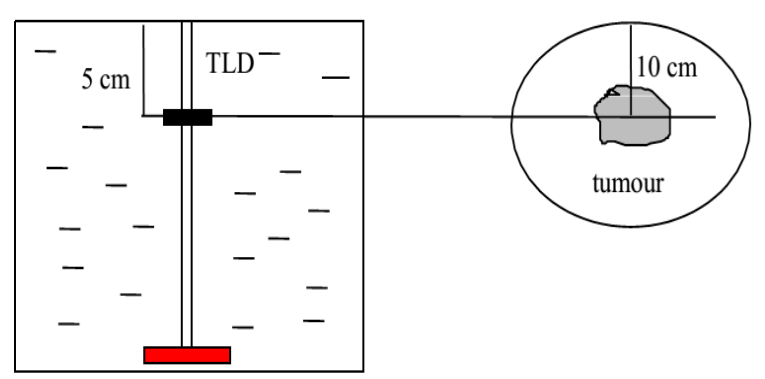

Figure 1: Irradiation geometry for the TLD and for a tumour. (Source: IAEA instruction sheet)

Another TLD capsule served as a background record. The irradiation took place under the conditions of the nominal source-to-axis distance $(\mathrm{SAD})$ used clinically at the centre, $10 \mathrm{~cm} \times 10 \mathrm{~cm}$ field size, $5 \mathrm{~cm}$ depth in water, and a $10 \mathrm{~cm} \times 10 \mathrm{~cm}$ field size. At first the IAEA recommended that the irradiation be done at $10 \mathrm{~cm}$ depth in water.

In the absorbed dose measurements, the calculation was done in accordance with the IAEA TRS 277 dosimetry protocol (IAEA, 1987) and TRS 398 dosimetry protocol (IAEA, 2000). Before 2002, the protocol used was the IAEA TRS 277. The calculation was made on the basis of the ionization chamber measurements, which was done at the point of the center of a TLD capsule, in the water phantom.

The TLDs and the Data Sheets were then returned to the agency within one week after the irradiation.
The \% deviation relative to IAEA measured dose was calculated according to the relation:

$$
\text { Deviation } \%=\frac{(X-Y)}{Y} * 100
$$

Where $\mathrm{X}$ is the stated dose from various centres and $\mathrm{Y}$ is the IAEA mean measured dose

\section{RESULTS AND DISCUSSIONS}

The results of Co-60 beam output checks performed by the National Centre for Radiotherapy and Nuclear Medicine, Korle-Bu for IAEA/WHO TLD postal programme from 1998 to 2012 are shown in Tables 2 and 3.

Table 1 : Results of the IAEA/WHO TLD postal dose audits of the National Centre for Radiotherapy and Nuclear Medicine Department, Korle-Bu. Each data point corresponds to the average of two TLDs.

\begin{tabular}{l|ccccccccc}
\hline $\begin{array}{l}\text { Year of } \\
\text { Audit }\end{array}$ & 1998 & 1998 & 2000 & 2002 & 2004 & 2006 & 2008 & 2010 & 2012 \\
\hline $\begin{array}{l}\text { Centre's } \\
\text { stated dose }\end{array}$ & 2.54 & 2.00 & 2.00 & 2.00 & 2.00 & 2.00 & 2.00 & 2.00 & 2.00 \\
$\begin{array}{l}\text { IAEA } \\
\text { mean dose }\end{array}$ & 2.06 & 2.09 & 2.07 & 1.99 & 2.02 & 2.04 & 2.08 & 2.07 & 2.00
\end{tabular}

Table 2 : Results of TLD measurements for ${ }^{60} \mathrm{Co}$ Beam. The \%deviation represents the \% deviation relative to IAEA mean dose.

\begin{tabular}{lccccccccc}
\hline Year of audit & 1998 & 1998 & 2000 & 2002 & 2004 & 2006 & 2008 & 2010 & 2012 \\
\hline \% Deviation & $\mathbf{2 3 . 5 0}$ & -4.30 & -3.30 & 0.50 & -1.2 & -2.00 & -3.90 & -3.50 & 0.10 \\
$\frac{\text { LAEA mean dose }}{\text { Centre's stated dose }}$ & 0.81 & 1.05 & 1.03 & 1.00 & 1.01 & 1.02 & 1.04 & 1.04 & 1.00 \\
\hline
\end{tabular}

In Table 1, data from the table correspond to absorbed dose measurements presented by the National Centre for Radiotherapy and Nuclear Medicine Department, and the IAEA. Each value point corresponds to the average of two TLDs. Table 2 presents all deviations, calculated according to the equation (1) for each year. The deviations exceeding the acceptable level of $\pm 5 \%$ is marked in bold. 


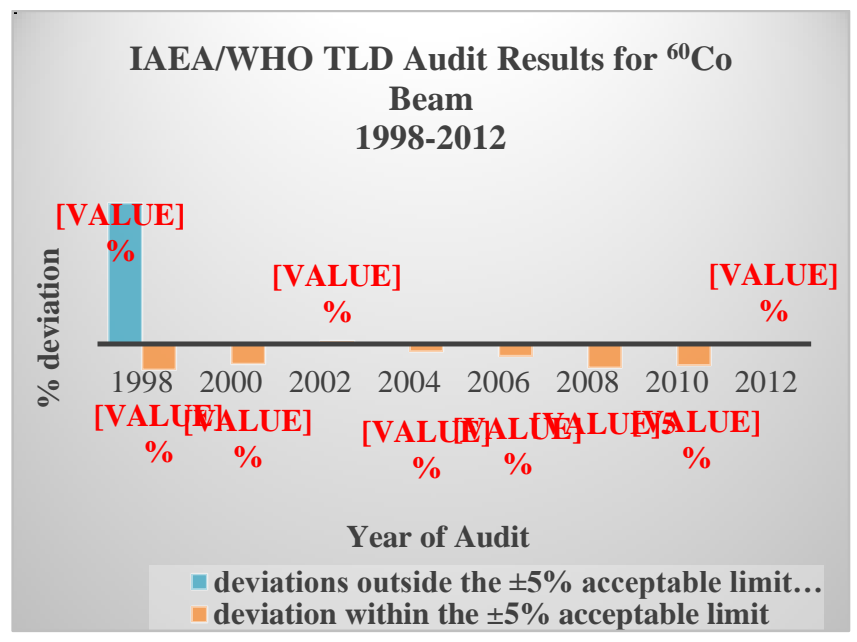

Figure 2: Results of the IAEA/WHO TLD postal dose audits of the National Centre for Radiotherapy and Nuclear Medicine Department, for the delivery of absorbed dose to water under reference conditions during $1998-2012$.

In Figure 2, the data point in the graph correspond to the \% deviation of the Centre's stated dose relative to the IAEA mean dose. The blue bar (for year-1998) corresponds to 1 st participation, and the second column (for year-1998) corresponds to the follow-up. The uncertainty in the TLD measurement of the dose is $1.8 \%$ ( 1 standard deviation). This does not include the uncertainty intrinsic to the dosimetry protocol. The deviations vary between a minimum $\%$ deviation of $0.1 \%$ and a maximum of $23.5 \%$.

Figure 3 also presents absorbed dose measurements made by the Centre, and the IAEA within the period of 1998 to 2012. Each data point in the graph corresponds to the average of two dosimeters.

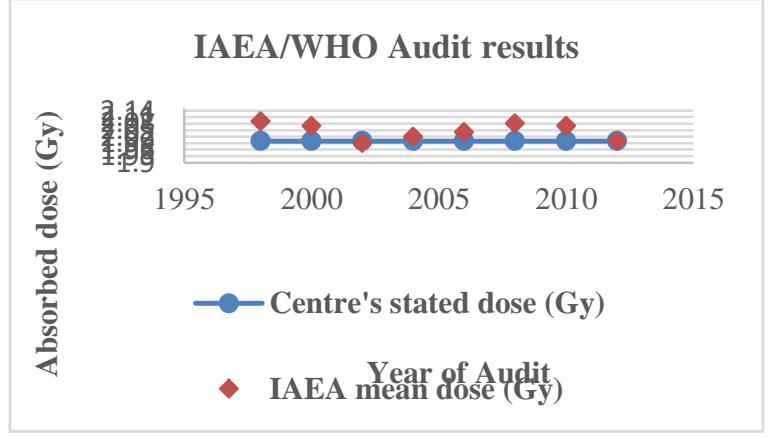

Figure 3 : Results of Absorbed Dose measurements from 1998 to 2012 (within the 5\% acceptable limit).

\section{IAEA TLD Audit Results for ${ }^{60} \mathrm{Co}$ Beam}

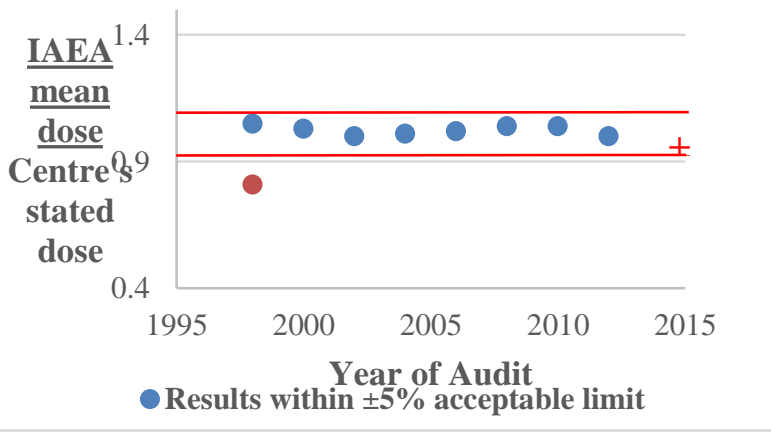

Figure 4 : Results of the IAEA/WHO TLD audits of the National Centre for Radiotherapy and Nuclear Medicine Department for the delivery of absorbed dose to water under reference conditions from 1998 to 2012.

The results from the entire period of study confirm the high standards in dosimetry and quality assurance, and further gives hope that such high standards may be maintained in the Centre.

The result outside the acceptable limit $\pm 5 \%$ was followed up by the agency. During the follow up, it was proven indubitably that deviation of $23.5 \%$ (first audit of Co-60 unit in the Centre) did not influence the patients. In that case, the errors were made during the audit. The physicist did not compare the output used for patient treatment with that reported in the TLD data sheet. The error was therefore explained and corrected during IAEA's follow up. 


\section{Conclusion}

The results of this study demonstrate that the QUATRO audit programme is a very useful tool for reviewing practices at our Centre, and shown beyond doubt to be effective in assuring the quality of dose determination at the Centre. Our study further indicates that it is possible to have the dose determination within acceptance limits by implementing correct procedures and cautiously performing dose calculations and measurements.

It is however recommended that training sessions be held for clinical physicists, so as to effectively and carefully adhere to the acceptable procedures of the IAEA dosimetry audits. With this in place, errors obtained during audits would be eradicated, leading to truly reflecting results on the Centre's radiotherapy practices.

\section{Acknowledgements}

The research team are grateful to physicists at the National Centre for Radiotherapy and Nuclear Medicine Department (KBTH) for their immense contribution in the various stages of this study. We also acknowledge the support of the Ghana Atomic Energy Commission (GAEC).

\section{REFERENCES}

[1]. Borras, C., 2006. Overexposure of radiation therapy patients in Panama: problem recognition and follow-up measures.

[2]. Groth, S., Meghzifene, A. \& Tatsuzaki, H., 2001. Quality assurance in radiotherapy. Proceedings of the International Conference on Radiological Protection of Patients in Diagnostic and Interventional Radiology, Nuclear Medicine and Radiotherapy.. pp. Pp. 618-624.
[3]. IAEA/WHO, 1997. SSDL NEWSLETTER. The Joint IAEAWHO Secretariat of SSDL network. ISSN 1011-2669, July.Issue 37.

[4]. IAEA/WHO, 2001. Scientific Committee of the IAEA/WHO Network of Secondary Standard Dosimetry Laboratory. Report of the. SSDL Newsletter, Issue 44, pp. Pp. 6-19.

[5]. IAEA, 1987. Absorbed dose determination in photon and electron beams. An international code of practice.. Issue 277.

[6]. IAEA, 1998. Accidental Overexposure of Radiotherapy Patients in San José. STI/PUB/1027.

[7]. IAEA, 2000. Absorbed Dose Determination in External Beam Radiotherapy. An International Code of Practice for Dosimetry Based on Standards of Absorbed Dose to Water. Technical Reports Series, Issue 398.

[8]. IAEA, 2000. Lessons learned from accidental Exposures in Radiotherapy. Safety Reports Series, Issue 17.

[9]. IAEA, n.d. s.l.: s.n.

[10]. ICRP, 2000. Prevention of Accidental Exposures to Patients. International Commission on Radiological Protection, Volume 86.

[11]. Izewska, J. \& Andreo, P., 2000. The IAEA/WHO TLD postal programme for radiotherapy hospitals Radiotherapy and Oncology. Issue 54, pp. Pp 65-72.

[12]. Izewska, J., Bera, P. \& Vatnitsky, S., 2002. IAEA/WHO TLD postal dose audit service and high precision measurements for radiotherapy level dosimetry Radiation Protection Dosimetry. pp. Pp. 387-392.

[13]. Izewska, J. et al., 2007. A methodology for TLD postal dosimetry audit of high-energy radiotherapy photon beams in non-reference conditions.. Radiother. Oncology 84 (1), pp. Pp. 67-74..

[14]. Izewska, J., Vatnitsky, S. \& Shortt, K. R., 2004. IAEA/WHO postal dose audits for radiotherapy 
hospitals in Eastern and South-Eastern Europe Cancer Radiothérapie. Issue 8, pp. Pp 36-43.

[15]. Waligorski, M. \& Lesiak, J., 2001. Radiological Protection of the Radiotherapy Patient? Proceedings of the. pp. Pp. 561-3.

[16]. WHO, 1988. Quality Assurance in Radiotherapy. organization, World Health. 